\title{
Chemical Profiling of Some Promising Black Tea Brands With Special Reference To Cup Quality
}

\author{
Ali Imran*1, Muhammad Umair Arshad ${ }^{1}$, Farhan Saeed ${ }^{1}$, Muhammad Sajid Arshad ${ }^{1}$, Muhammad Imran ${ }^{2}$, \\ Muhammad Nadeem ${ }^{3}$, Aftab Ahmed ${ }^{1}$, Usman Naeem ${ }^{1}$,Muhammad Arslan Aslam ${ }^{1}$, Sara Isthaq ${ }^{1}$ and Darosham \\ Sohail Khan ${ }^{1}$
}

${ }^{1}$ Institute of Home \& Food sciences, Government College University, Pakistan

${ }^{2}$ Department of Allied Health Sciences, Imperial College for Biological Studies, Pakistan

${ }^{3}$ Department of Environmental Sciences, COMSATS Institute of Information Technology (CIIT), Vehari-Pakistan

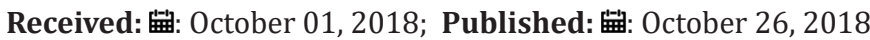

*Corresponding author: AliImran, Assistant Professor, Institute of Home \& Food Sciences, GCUF, Faisalabad, Pakistan

\begin{abstract}
Present research was conducted in different available tea brands for exploring their phytochemical profiling with special consideration of tea cup quality. Research design was based upon the extraction of antioxidant with varied concentration of methanol and optimization of extraction criteria. Higher extraction yield was noted for 20 minutes as compared to 10 minutes. As a function of extraction time, various tea quality parameters were increased. The values regarding cup quality indicated that T1 (commercial brand 1) hold highest theaflavin as 1.84\% in contrast with T4 (loose pack) that contained lowest as $1.78 \%$. Whereas, T5 had highest level of theabronin (20.23\%) indicating strong color and brightness of the extract but lessen its allied health benefits. In general tea extract, which was under study, exhibited good antioxidant and free radical scavenging activity ranging from 39.36 to 44.87 and 47.56-70.01\%, respectively. Caffeine content was seemed to be in safe limit (1.17- 1.39\%).
\end{abstract}

Keywords: Theaflavin; Thearubingins; Cup Quality; Methanolic Extracts; Purine Allkolids

Abbreviations: TFs : Theaflavins; EI : Ethanolic Solution; TPC: Total Phenolic Contents; Completely CRD: Randomized Design; LSD: Least Significance Test

\section{Introduction}

Enhanced association between nutrition and health with inclination to avoid maladies rather than cure and concept of diet-based therapy have led to the concept of nutraceuticals. Now nutraceuticals are considered as a mainstay in domain of dietbased therapies. Phytochemicals are in attention for a long time for discoveries of the nutraceuticals [1] Researchers and scientists divert their attention towards the discoveries of those phytochemicals that are used in daily diet as well as not expensive. In that sense certain discoveries are appeared in the horizon of nutraceuticals and black tea and its constituents is one of the important components of these strategies to mitigate and cure various diseases primarily due to its antioxidant potential. Tea brewed from the plant Camellia sainensis is consumed in different parts of the world as green, black or oolong tea. However, black tea occupies about $80 \%$ of the world tea production, while rest is contributed by green and oolong tea $[2,3]$. During the production of black tea, about $75 \%$ of major polyphenols of the tea (catechins) undergo enzymatic transformation consisting of oxidation and partial polymerization by polyphenoloxidize that catalyze the reaction and transforming tea inheriting antioxidant into distinguished black tea compounds, nevertheless some can remain as an original state as catechins.

The resulting black tea composition depends upon the technological procedures [3]. Black tea pytochemistry like the other medicinal plant also inheriting with some prominent constitute but also accompanied by some components that proves a thereat when used in an excessive amount. Major components are catechins, theaflavins, thearubigins, caffeine and flavonols Bech-Larsen, Scholderer, et al. Black tea parental composition is strongly resembles with other teas but its divine components make it an alternative source for incorporation into diet based therapies [4]. Major polyphenols in black tea are theaflavins, Thearubingins and theabronin along with unoxidized catechins [5]. Theaflavins (TFs) are dimeric polyphenols produced during production of tea. Due to strong antioxidant properties, there is growing interest in 
the food, beverages and healthcare industries in producing and incorporation of theaflavins in various products [6], produced during the oxidative fermentation of tea leaf. Thearubingins (TRs) are the high molecular weight compounds that are responsible for the tea brisk and astringent and present almost in double amount as the theaflavins [7]. They also give body to the brewed tea.

TRs are larger in size and darker in color than TFs [1]. TRs are a heterogeneous group of phenolic pigments with relative molecular masses in the range 700-40,000 $\mathrm{Da}$.The content of TFs in black tea is $0.3-2 \%$ on dry weight basis whilest the TRs fraction comprises $10-20 \%$ of black teas and together with TFs contribute to the tea brew characteristics such as color, strength and body [8]. Black tea flavonols included quercetin, kaempferol and myricetin. They make up $2-3 \%$ of the water-soluble extract in tea. These flavonol have proved effective against cancer and also inhibit the growth of malignant cell [9-11]. Cup quality of tea is referred by the amount of selected parameters that contributed the pharmacological and health associated properties of the tea [12]. Health benefits of tea cannot be understood without polyphenols. Tea polyphenols are associated with some key health benefits like anticancer, anti-mutagenesis and antidiabetic properties. Moreover, they are effectively used for the treatment of obesity, artherocesrolises and heart diseases [13].

In the guide line of theses finding there is a possibility for cup quality with the use of polyphenolic assay and other related attributes like total phenolic, antioxidant activity, theaflavin, therubingin and theabronins along with total catechins and caffeine can effectively describe the cup quality [14]. Tea active ingredient extraction is very crucial stage so optimization of extraction condition is a key factor [13]. The extraction process can be carried out by using different solvents like water, methanol, ethanol, and acetonitrle. Water exhibited least extraction yield as contract to others [14]. Time and temperature have a great importance in this contest, generally increase in time and temperature proved beneficial for extraction efficiency. Multi-step extraction with gradually increase time yields better as compared to single step extraction. Extraction decreased when it extended prolonged in a single step due to the destruction of the poly phenols [15]. Present research was carried out to explore the extraction efficiency of polyphenols with respect to the time variation using methanol as solvent and it is correlated with tea cup quality to set its role in the modern life style.

\section{Material and Method \\ Procurement of Samples}

Different commercial brands of black tea i.e. paper packed, loose and tea bags were procured from the local market (Table 1). Reagents and standards were purchased from Sigma-Aldrich (Sigma-Aldrich Tokyo, Japan) and Merck (Merck KGaA, Darmstadt, Germany). The collected tea samples were stored at ambient temperature for further study.

Table 1: Treatment used in the study.

\begin{tabular}{|c|c|c|c|}
\hline Extract name & Description & \multicolumn{2}{c|}{ Extraction condition } \\
\hline $\mathrm{T}_{1}$ & Commercial brand 1 & Time 10 and 20 minutes & Solvent=methanol \\
\hline $\mathrm{T}_{2}$ & Commercial brand 2 & Solvent=methanol & Time 10 and 20 minutes 10 and 20 minutes \\
\hline $\mathrm{T}_{3}$ & Commercial brand 3 & Solvent=methanol & Time 10 and 20 minutes \\
\hline $\mathrm{T}_{4}$ & Loose tea & Solvent=methanol & Time 10 and 20 minutes \\
\hline $\mathrm{T}_{5}$ & Tea bag 1 & Tolvent=methanol \\
\hline $\mathrm{T}_{6}$ & & Time 10 and 20 minutes 2 & \\
\hline
\end{tabular}

\section{Preparation of Tea Extracts}

Tea brands were subjected to extraction by using methanol as solvent according to specified method given by [14]. Tea sample $(5 \mathrm{~g})$ from each brand was extracted with methanol $(100 \mathrm{~mL})$ on a water bath at $60^{\circ} \mathrm{C}$ for 10 and 20 minutes. Extracts were filtered by using vacuum filtration assembly and solvent was recovered by rotary vacuum evaporator (EYELA, N-N series, Japan) at $40^{\circ} \mathrm{C}$. The extract of each sample was weighted to determine the yield of soluble constituents and stored at $4^{\circ} \mathrm{C}$ until further use.

\section{Analysis of Tea Extracts}

Tea extracts were analyzed for their antioxidant potential for indices like total phenolics, antioxidant activity and free radical scavenging activity (DPPH assay) by following respective procedures. The brief description of each method is given below.

\section{Total Phenolics}

Tea solution $(1 \mathrm{~mL})$ was taken into a volumetric flask then added $4 \mathrm{~mL}$ distilled water and $5 \mathrm{~mL}$ of tartrate solution $\left(1 \mathrm{~g}\right.$ of $\mathrm{FeSO}_{4}$ and $5 \mathrm{~g}$ of $\mathrm{KNaC}_{4} \mathrm{H}_{4} \mathrm{O}_{6}$ dissolved in $1000 \mathrm{~mL}$ distilled water). It was diluted with $25 \mathrm{~mL}$ buffer solution $\left(23.377 \mathrm{~g}\right.$ of $\mathrm{Na}_{2} \mathrm{HPO}_{4}$ in $1000 \mathrm{~mL}$ of distilled water and $9.078 \mathrm{~g}$ of $\mathrm{KH}_{2} \mathrm{PO}_{4}$ in $1000 \mathrm{~mL}$ of distilled water in the ratio of $85 \%$ and $15 \% \mathrm{v} / \mathrm{v}$ ). The absorbance was measured using UV/Vis spectrophotometer (CESIL CE7200, Japan) at $540 \mathrm{~nm}$ as described by [14].The calculation was carried out by using following expression. Total polyphenols $(\%)=0.3914 \mathrm{EV0} /$ V1/W [15-17].

\section{Where as \\ $\mathrm{E}=$ The absorbance reading of the spectrophotometer \\ V0 $=$ Total volume of the tea solution $(250 \mathrm{~mL})$}


$\mathrm{V} 1$ = Volume used for the measurement $(1 \mathrm{~mL})$

$\mathrm{W}=$ The dry weight of the tea sample.

\section{Total Catechins}

The sample $(0.1 \mathrm{~mL})$ was taken in a test tube containing methanol $(0.9 \mathrm{~mL})$; transfer $2.5 \mathrm{~mL}$ of vanillin solution (1\% methanol) along with $10 \mathrm{~mL} 9 \mathrm{~N} \mathrm{HCl}$ in the same tube. The solution was kept for 30 minutes before measuring with UV/Vis spectrophotometer at $500 \mathrm{~nm}$ against the corresponding reagent methanol as blank [18].

\section{Antioxidant Activity}

$\beta$-carotene $(2 \mathrm{mg}$ ) was dissolved in $20 \mathrm{~mL}$ chloroform. Aliquot of $(3 \mathrm{~mL})$ solution was transferred into $50 \mathrm{~mL}$ beaker and $40 \mathrm{mg}$ linoleic acid and 400mg Tween 20 were added. Chloroform was removed by purging with nitrogen. Oxygenated distilled water $(100 \mathrm{~mL})$ generated by bubbling air into distilled water for 1 hour was added in $ß$-carotene emulsion and mixed well by using vortex mixer. Aliquots $(3 \mathrm{~mL})$ of the oxygenated $ß$-carotene emulsion and $0.10 \mathrm{~mL}$ of phenolic extracts were placed in capped culture tubes and mixed vigorously. The tubes were immediately placed in a water bath and incubated at $50^{\circ} \mathrm{C}$. Oxidation of $ß$-carotene emulsion was monitored spectrophotometrically by measuring absorbance at $470 \mathrm{~nm}$ after $0,10,20,30$ and 40 minutes as indicated by [16].The degradation rate of the extracts was calculated according to first order kinetics using following equation

$$
\begin{aligned}
& \text { In }(\mathrm{a} / \mathrm{b}) \times 1 / \mathrm{t}=\text { sample degradation rate } \\
& \ln =\text { The natural } \log \\
& \mathrm{a}=\text { The initial absorbance }(470 \mathrm{~nm}) \text { at time zero } \\
& \mathrm{b}=\text { The absorbance }(470 \mathrm{~nm}) \text { after } 40 \mathrm{~min} \\
& \mathrm{t}=\text { The time }(\mathrm{min})
\end{aligned}
$$

\section{Free Radical Scavenging Activity (DPPH assay)}

Extract solutions were prepared by dissolving $0.025 \mathrm{~g}$ of dry extract in $10 \mathrm{~mL}$ of ethanol. A fresh solution of DPPH• in ethanol (6×10-5 M) was prepared before measurement. Approximately $3 \mathrm{~mL}$ of solution was mixed with $77 \mu \mathrm{L}$ (38 or $19 \mu \mathrm{L}$ in additional assays) extract solution in $1 \mathrm{~cm}$ path length disposable microcuvette. The samples were kept in dark for 15 minutes at room temperature; the decrease in absorbance was measured at $515 \mathrm{~nm}$. Absorbance of blank sample containing the same amount of ethanol and DPPH• solution was also measured in the same fashion as describe by [17]. Radical scavenging activity was calculated by the following formula

$$
\begin{aligned}
& \text { Reduction of absorbance }(\%)=[(\mathrm{AB}-\mathrm{AA}) / \mathrm{AB}] \times 100 \\
& \mathrm{AB}=\text { Absorbance of blank sample }(\mathrm{t}=0 \mathrm{~min}) \\
& \mathrm{AA}=\text { Absorbance of tested extract solution }(\mathrm{t}=15 \mathrm{~min})
\end{aligned}
$$

\section{Tea Quality Parameters}

Tea quality parameters like theaflavin, theabrownin and thearubingins were determined according to their respective method (Muralidharan) [14] Extracts were treated with different ethanolic treatments namely EI, EII, EIII and EIV as described below
Ethanolic Solution I (EI): Tea solution ( $30 \mathrm{~mL})$ was mixed with ethyl acetate $(30 \mathrm{~mL})$ in a separating funnel, shake for 5 minutes. Part of the ethyl acetate layer $(2 \mathrm{~mL})$ was diluted to $25 \mathrm{~mL}$ with $95 \%$ $(\mathrm{v} / \mathrm{v})$ ethanol. The absorption of ethanol solution was recorded as EI.

Ethanolic Solution II (EII): A portion of original tea solution $(15 \mathrm{~mL})$ was mixed with n-butanol $(15 \mathrm{~mL})$ followed by shaking for 3 minutes. Part of the aqueous layer $(2 \mathrm{~mL})$ was mixed with saturated oxalic acid $(2 \mathrm{~mL})$ and distilled water $(6 \mathrm{~mL})$ and then diluted with $25 \mathrm{~mL}(95 \% \mathrm{v} / \mathrm{v}$ ) ethanol. The absorption of ethanol solution was recorded as EII.

Ethanolic Solution III (EIII): 15mL of the ethyl acetate layer was mixed with equal amount of $(2.5 \% \mathrm{w} / \mathrm{v}) \mathrm{NaHCO}_{3}$ followed by shaking for $30 \mathrm{sec}$. The aqueous layer was discarded and part of ethyl acetate layer $(4 \mathrm{~mL})$ was diluted with $25 \mathrm{~mL}$ ethanol. The absorption of ethanol solution was recorded as EIII.

Ethanolic Solution IV (E IV): Aqueous layer of Ea ( $2 \mathrm{~mL})$ was mixed with saturated oxalic acid ( $2 \mathrm{~mL})$ and distilled water $(6 \mathrm{~mL})$ and diluted with $25 \mathrm{ml}(95 \% \mathrm{v} / \mathrm{v})$ ethanol. The absorption of this ethanol solution was recorded as EIV at $380 \mathrm{~nm}$.

The value of specific tea quality parameters was calculated by following expression. Theaflavin $\%=\mathrm{TF} \% 2.25 \mathrm{EIII} / 1-\mathrm{M}$

Thearubingin $\%=$ TR\% 7.06 (2EI+2EIV-2EII-EIII) $/ 1-\mathrm{M}$

Theabronin \% = TB\% 7.06*2Eb/1-M

Where EI, EII, EIII and EIV are the corresponding (absorbance) readings taken with spectrophotometer, whereas, $\mathrm{M}$ is the moisture content of the tea sample

\section{Caffeine Determination}

Caffeine was determined in tea samples following the method of [17]. An accurately amount of sieved tea (50mg) was dissolved in $(25 \mathrm{~mL})$ of distilled water. The solution was stirred for one hour using magnetic stirrer and heated gently to remove caffeine from the solution. Finally, the absorbance of the solution was measured at $310 \mathrm{~nm}$ against the corresponding blank.

\section{Statistical Analysis}

Data obtained for each parameter was subjected to statistical analysis by applying completely randomized design (CRD) using Statistical Package (Statixtic V-8.1). Significant ranges were further compared using least significance test (LSD) [18].

\section{Result and Discussion}

\section{Extraction Efficiency}

Choice for selecting an extraction method based its ability to preserve the bioactive compounds and extract most of these otherwise at least if not all the desired compounds. Wet extractions involve solid material in direct contact with a liquid solvent [19]. During the extraction, organic solvents diffuse into the solid material and solubilize compounds with similar polarity. The nature of the solvent used will determine the types of chemicals likely to be extracted from the plant. Organic solvents for extractions include 
polar solvents such as water, ethanol, methanol and acetone to nonpolar solvents such as dichloromethane and hexane.

The extraction yield differs significantly with respect to extraction time. The results regarding the means (Table 2) for extraction yield varies from 21.3 to $25.7 \%$ for 10 minutes extraction time. T5 (tea bag 1) showed maximum yield (25.7\%) while T1 that was the commercial packed 1 exhibited the minimum yield $(21.3 \%)$. Extraction yield increased prominently when extraction time was increased. The yield for 20 minutes extracts was varied between 25 to $31 \%$ and maximum found in T5 (TB1) as 31\%. Extraction efficiency was increased by increase the time of extraction however, percent extraction depend on type of solvent [14]. In another study it was found that methanol was an effective solvent for the extraction of antioxidant [19].

Table 2: Mean for extraction yield of various tea brands using methanol as solvent for 10- and 20-minutes extraction time.

\begin{tabular}{|c|c|c|}
\hline Treatment & Methanol $_{10}(\%)$ & Methanol $_{20}(\%)$ \\
\hline $\mathrm{T}_{1}$ & $21.3 \bigotimes 1.5 c$ & $25.3 \bowtie 0.58 \mathrm{a}$ \\
\hline $\mathrm{T}_{2}$ & $23.3 \otimes 1.5 \mathrm{c}$ & $28.3 \bowtie 0.58 \mathrm{a}$ \\
\hline $\mathrm{T}_{3}$ & $24.0 \rrbracket 4.4 \mathrm{~b}$ & $28.0 \bowtie 1.00 \mathrm{a}$ \\
\hline $\mathrm{T}_{4}$ & $24.0 \bigotimes 1.0 \mathrm{~b}$ & $25.0 \bigotimes 1.00 \mathrm{a}$ \\
\hline $\mathrm{T}_{5}$ & $25.7 \otimes 4.5 \mathrm{a}$ & $31.0 \otimes 1.00 \mathrm{a}$ \\
\hline $\mathrm{T}_{6}$ & $24.7 \rrbracket 3.5 a$ & $30.3 \bowtie 0.58 \mathrm{a}$ \\
\hline
\end{tabular}

Note: Values are mean+ sd

Methanol $_{10}(\%)$ methanol at 10 minutes extraction time.

Methanol $_{20}(\%)$ methanol at 20 minutes extraction time.

\section{Total Phenolic Contents (TPC)}

Phenolics and polyphenolic compounds constitute the main class of natural antioxidants present in plants and may contribute directly to antioxidative action [20]. Means for TPC depicted (Table 3) that extract ranged from $39.36 \%$ to $44.87 \%$. T2 (CB2) commercial brand 2 showed the highest (44.87\%) TPC contents whilst, lowest was exhibited by T4 (LP) loose pack as $39.36 \%$ of 10 minutes extracts. Highest value for 20 minutes extract (44.95\%) in T2 (CB2) followed by T1 (CB1) as $42.36 \%$. The lowest amount (40.51\%) was recorded in T4 (LP). This is might be due to the presence of more moisture as compared to the others. In another study total phenolic contents of different tea samples were found to be in the range of 14 to $45 \%$, highlighted the effect of extraction time and solvent. This increase in the TPC contents emphasize the importance of solvent and methanol proved better from water probably due to its nature [21]. Another study conducted in Australian supermarket on different tea samples with water showed the total polyphenols in black teas ranged from $14 \%$ to $34 \%$, in of different brands extracted with water these differences are might be due to solvent, extraction time and temperature Wright et al. A hypothesis was developed by [22] explained the role of temperature and time in the extraction that was proved true by the [14] ;extraction time is a key factor for determination the extraction efficiency which effect all its attributes

\section{Antioxidant activity}

Antioxidant activity of different tea extract of 10 minutes extraction time revealed (Table 3) that T1 (CB1) showed the highest amount for that trait (52.02\%) followed by T2 (CB2) as $49.39 \%$. The lowest amounts (40.02\%) was noted in T6 (TB2). The same relation was found in extracts of 20 minutes among them T1 (CB1) showed the highest antioxidant activity (54.02\%) followed by T2 (CB2) as 49.39\%. The lowest activity (40.04\%) was recorded in T6 (TB2). Extraction for antioxidant activity from tea was dependent upon the choice of solvent and methanol was found to be beneficial in this regard [22]. also found the antioxidant activity of black tea methanolic extracts at higher time gives higher antioxidant activity as compared with water and methanol at shorter times. In an early study, the antioxidant status of different tea samples were estimated and found that methanolic extracts of green, oolong and black tea showed antioxidant activity ranged from 50 to $80 \%$ accordingly green tea exhibited maximum activity [19].

Table 3: Mean values of some promising characteristic of tea extracts.

\begin{tabular}{|c|c|c|c|c|c|c|}
\hline \multirow[t]{2}{*}{ Treatment } & \multicolumn{2}{|c|}{ ТPC (\%) } & \multicolumn{2}{|c|}{ Beta Carotene (\%) } & \multicolumn{2}{|c|}{ DPPH Activity (\%) } \\
\hline & Methanol $_{10}$ & Methanol $_{20}$ & Methanol $_{10}$ & Methanol $_{20}$ & Methanol $_{10}$ & Methanol $_{20}$ \\
\hline $\mathrm{T}_{1}$ & $42.35 \llbracket 0.01 \mathrm{~b}$ & $42.36 \bowtie 0 \mathrm{~b}$ & $52.02 \bowtie 0.01 \mathrm{a}$ & $54.02 \bowtie 0.01 \mathrm{a}$ & $55.61 \bowtie 0.49 \mathrm{~b}$ & $68.42 \bowtie 0.01 \mathrm{ab}$ \\
\hline $\mathrm{T}_{2}$ & $44.87 \llbracket 0.01 \mathrm{a}$ & $44.95 \bowtie 0.01 \mathrm{a}$ & $49.39 \llbracket 0.5 \mathrm{~b}$ & $50.01 \llbracket 0.06 \mathrm{bc}$ & $59.11 \bowtie 0.02 \mathrm{a}$ & $68.42 \llbracket 0.01 \mathrm{ab}$ \\
\hline $\mathrm{T}_{3}$ & $40.03 \llbracket 0.01 \mathrm{e}$ & $41.38 \llbracket 0.01 \mathrm{~d}$ & 45.00区.01c & $49.0 \otimes 0.05 \mathrm{~d}$ & $55.05 \llbracket 0.02 \mathrm{c}$ & $70.01 \bowtie 0.02 \mathrm{a}$ \\
\hline $\mathrm{T}_{4}$ & $39.36 \varangle 0.05 \mathrm{~d}$ & $40.51 \bowtie 0.01 \mathrm{c}$ & $46.03 \bowtie 0.05 \mathrm{~d}$ & $50.00 \varangle 0.01 \mathrm{c}$ & $54.06 \varangle 0.01 \mathrm{~d}$ & $66.40 \rrbracket 2.83 \mathrm{~b}$ \\
\hline $\mathrm{T}_{5}$ & $40.41 \rrbracket 0.06 \mathrm{~d}$ & $41.38 \bowtie 0.01 \mathrm{f}$ & $47.01 \rrbracket 0.01 \mathrm{e}$ & $50.05 \llbracket 0.05 \mathrm{~b}$ & $47.56 \llbracket 0.16 \mathrm{a}$ & $62.05 \bigotimes 1.67 \mathrm{c}$ \\
\hline $\mathrm{T}_{6}$ & $42.15 \rrbracket 0.05 \mathrm{c}$ & $41.72 \llbracket 0.04 \mathrm{e}$ & $40.02 \llbracket 0.01 \mathrm{f}$ & $45.04 \llbracket 0.01 \mathrm{e}$ & $54.04 \llbracket 0.03 \mathrm{~d}$ & $60.48 \bowtie 0.46 \mathrm{c}$ \\
\hline
\end{tabular}

Note: values are mean+

\section{DPPH Free Radical Scavenging Activity}

It is one of the stable and commercially available organic radicals bearing no similarity to the highly reactive and transient peroxyl radicals involved in various oxidative reactions in vivo. DPPH free radical scavenging activity indicates antioxidant potential of selected bioactive molecules [23]. Mean for DPPH free radical scavenging activity of different tea brands at 10 minutes extracts (Table 3) ranged from 47.56 to $59.11 \%$. Maximum free radical inhibition off $59.11 \%$ in T2 (CB2) followed by $55.6 \%$ in $\mathrm{T} 1$ (CB1) and minimum was observed as $47.56 \%$ in T5 (TB1). While means for 20 minutes extracts ranged from 60.48 to $70.01 \%$. 
Maximum inhibition $70.01 \%$ was found in T3 (CB3) followed by $68.42 \%$ in T1 (CB1) while the minimum activity was found $60.48 \%$ in T6 (TB2). A project that was conducted for evaluation of time and temperature for extracting the antioxidant and it was revealed that enhanced temperature and time implicit a positive effect for that trait. Methanol used as solvent for that regards in another study and found that work better than the other $[19,22]$.

\section{Cup Quality}

Cup quality reflects health promoting parameters of the tea in the single serving of the cup. The quality of tea brands highly depend upon this parameters. This is a healthy attribute that determined the quality of the brand. Tea bioactive molecules are theaflavin, thearubingin, theabronin and total catechins as well as the caffeine. The brief discussion concerning these parameters is described below.

\section{Theaflavin}

Theaflavin contributed towards the taste, brisk and astringent to the tea along with bright golden color. Theaflavin levels generally depend upon the amount of fermentation, genetic variations and climatic condition of the growing region so its level varied in the different cultivator. Mean for theaflavin in commercially available brands in Pakistan (Table 4) showed highest level (1.83\%) in T1 (CB1) whereas lowest (1.71\%) was detected in T4 (Lp) in 10 minutes extraction time. Results for 20 minutes extracts followed the similar trend like the earlier extraction condition with enhanced yield. Maximum recovery of theaflavin was recorded in T1 as $1.84 \%$ in contrast with T4 that yielded minimum (1.80\%). Highest level of theaflavin in $\mathrm{T} 1$ reflects its bright color and full fermentation, which is co-related with its strong taste. However, theaflavin amount also dependent upon initial moisture content. The effect of extraction temperature exhibited a pronounced effect upon solubilization of theaflavin. Extraction time along with temperature is a prime factor affecting the concentration of theaflavin in the final extracts. Lowest level in T4 might be due to its packaging condition loose packaging caused more moisture content that result in low Theaflavin contents. Another reason might be due to over fermentation of the samples that was required by their specific buyer. Difference in the Theaflavin contents may be due to the difference in their permeability that proved a driving forced for extracting this active compound Theaflavin from them.

Table 4: Means for Theaflavin, thearubingins and total catechin contents (\%) of methanolic extracts at 10 and 20 minutes extraction time.

\begin{tabular}{|c|c|c|c|c|c|c|}
\hline \multirow[t]{2}{*}{ Treatment } & \multicolumn{2}{|c|}{ Theaflavin contents (\%) } & \multicolumn{2}{|c|}{ Thearubingins Contents (\%) } & \multicolumn{2}{|c|}{ Total catechins contents (\%) } \\
\hline & Methanol $_{10}$ & Methanol $_{20}$ & Methanol $_{10}$ & Methanol $_{20}$ & Methanol $_{10}$ & Methanol $_{20}$ \\
\hline $\mathrm{T}_{1}$ & $1.83 \bowtie 0.01 \mathrm{~A}$ & $1.84 \llbracket 0.01 \mathrm{ab}$ & $1.81 \rrbracket 0.01 \mathrm{~cd}$ & $1.61 \rrbracket 0.3 \mathrm{a}$ & $1.29 \bowtie 0.05 \mathrm{c}$ & $1.36 \rrbracket 0.05 b$ \\
\hline $\mathrm{T}_{2}$ & $1.78 \bowtie 0.01 \mathrm{~A}$ & $1.82 \bowtie 0.01 \mathrm{ab}$ & $1.87 \bowtie 0.02 \mathrm{~cd}$ & $1.89 \bowtie 0.02 \mathrm{a}$ & $1.30 \otimes 0.01 \mathrm{~b}$ & $1.35 \bowtie 0.01 \mathrm{a}$ \\
\hline $\mathrm{T}_{3}$ & $1.77 \bowtie 0.01 \mathrm{~A}$ & $1.81 \rrbracket 0.01 \mathrm{ab}$ & $1.83 \llbracket 0.03 \mathrm{bc}$ & $1.80 \rrbracket 0.01 \mathrm{a}$ & $1.29 \bowtie 0.01 \mathrm{bc}$ & $1.31 \bowtie 0.01 \mathrm{ab}$ \\
\hline $\mathrm{T}_{4}$ & $1.71 \otimes 0.02 \mathrm{~A}$ & $1.80 \bowtie 0.01 \mathrm{~b}$ & $1.82 \bowtie 0.01 \mathrm{a}$ & $1.87 \rrbracket 0.01 \mathrm{a}$ & $1.28 \bowtie 0.01 \mathrm{a}$ & $1.31 \otimes 0.01 \mathrm{ab}$ \\
\hline $\mathrm{T}_{5}$ & $1.78 \bowtie 0.01 \mathrm{~B}$ & $1.83 \bowtie 0.03 a$ & $1.78 \bowtie 0.05 \mathrm{~d}$ & $1.78 \varangle 0.03 \mathrm{a}$ & $1.30 \otimes 0.01 \mathrm{c}$ & $1.30 \rrbracket 0.01 \mathrm{ab}$ \\
\hline $\mathrm{T}_{6}$ & $1.76 \bowtie 0.03 \mathrm{~A}$ & $1.81 \llbracket 0.01 \mathrm{ab}$ & $1.84 \bowtie 0.05 \mathrm{e}$ & $1.84 \llbracket 0.01 \mathrm{a}$ & $1.27 \bowtie 0.01 \mathrm{~d}$ & $1.32 \varangle 0.011 \mathrm{c}$ \\
\hline
\end{tabular}

Note: values are mean+ sd

Low TF content in black tea may be due to over-fermenting or long periods of storage [24]. The information derived from the earlier study used to predicting black tea country of origin based on polyphenols composition because geographical area of tea production may contribute to the pattern of individual theaflavin composition in black tea [25]. In another study it was proved that the theaflavin holds a strong effect on color and brightness of the tea more over, it was also suggested that the liquor brightness was due to the theaflavin and their constitute so it was noteworthy to quantification of theaflavins or enrichment for the production of the tea of strong taste and color Ali et al. Solvent extraction with methanol for 20 minute proved better then water but effect of temperature cannot be denied as well as time of extraction holds significant position in this contest so extraction time increase exhibited marked increase in their extraction efficiency as compared with that water and methanol for 10 minute. The influence of extraction time on extraction efficiency was observed in earlier study that was designed to determine the effect of varying temperature on the methanolic extraction. Results depicted the enhanced yield as the time of extraction was increased for theaflavin thearubingins and theabrownin as compared to shorter time. The ranged of $30 \%$ at shorter time while $33 \%$ at longer time was observed [1].

\section{Thearubingins}

Thearubingins are a group of compounds formerly recognized as insoluble fractions, SI and SII, of ethyl acetate extraction. TR was further separated by butanol into soluble and insoluble TR that contributed the reddish colour and richness in taste, totally termed as body to black tea. Thereby, it is associated with quality and other desirable liquor characters [26]. Results regarding the means for methanolic extracts at 10 min extraction time (Table 4 ) indicating the highest thearubingin value (1.87\%) in T2 (CB2) while, lowest $(1.78 \%)$ in T5 (TB1). In contrary, when extraction time was increased it reflected the maximum amount in $\mathrm{T} 2$ as $1.89 \%$ and minimum $(1.78 \%)$ in T5 (TB1). These result suggested the suitable temperature along with time of extraction were an influenced factors for extracting the tea active molecules. Highest level containing extract showed less brightness and darker look with contrast to extract containing fewer amounts Martin et al. 
proved a relation between liquor brightness and thearubingins and they inference a hypothesis that high level of thearubingins reduce the tea brightness. They also performed a hedonic evaluation for authenticity for this possibility proved that relation successfully.

\section{Theabronin}

Theabronin is a member of purine allkolids along with caffeine. Generally the amount of theabronin should be less in good quality tea. The mean values for the theabronin (Table 5) showed the same trend that its value increased when extraction time was increased. The extracts of 10 minutes varies from 17.06-19.70\% and heights claimed by $\mathrm{T} 1$ (CB1) as $19.70 \%$ followed by $\mathrm{T} 2$ as $18.58 \%$ and lowest depicted in T3 (CB3) as17.06\%. The maximum theabronin
(21.22 \%) was found in T5 (TB1\%) and minimum was reflected in T4 (LP) as $18.10 \%$. The amount of theabronin has a negative effect on the tea. TB endows tea liquor and leaf with a dark brown color, which has a negative effect on tea quality. The information that derived from analysis of theabronin used as objective method in the determination of black tea quality [25]. The extracts that contain the heights level of theabronin exhibited dark red color and strong appearance that was misleading with the quality. The highest level may be due to the origin, cultivar, climatic conditions and processing properties Amount of the theabronin and caffeine is a function of the extraction conditions and solvent used for tea active ingredient extraction. There is positive correlation for methanolic extraction as compared to the other solvents [27].

Table 5: Means for purine allkolids (caffeine and theabrwonin) content (\%) of extracts at 10 and 20 miutes extraction time.

\begin{tabular}{|c|c|c|c|c|}
\hline \multirow[t]{2}{*}{ Treatment } & \multicolumn{2}{|c|}{ Caffeine contents $(\%)$} & \multicolumn{2}{|c|}{ Theabrownin contents (\%) } \\
\hline & Methanol $_{10}$ & Methanol $_{20}$ & Methanol $_{10}$ & Methanol $_{20}$ \\
\hline $\mathrm{T}_{1}$ & $1.28 \llbracket 0.02 \mathrm{a}$ & $1.28 \otimes 0.02 \mathrm{~b}$ & $19.70 \bowtie 0.2 \mathrm{~b}$ & $20.28 \llbracket 0.76 \mathrm{~b}$ \\
\hline $\mathrm{T}_{2}$ & $1.2 \llbracket 0.09 \mathrm{ab}$ & $1.34 \bowtie 0.02 \mathrm{a}$ & $18.58 \bowtie 0.5 \mathrm{c}$ & $19.22 \bowtie 0.01 \mathrm{c}$ \\
\hline $\mathrm{T}_{3}$ & $1.21 \rrbracket 0.05 \mathrm{ab}$ & $1.18 \bowtie 0.02 \mathrm{c}$ & $17.06 \rrbracket 0.03 \mathrm{~d}$ & $18.08 \bowtie 0.02 \mathrm{~d}$ \\
\hline $\mathrm{T}_{4}$ & $1.17 \rrbracket 0.02 \mathrm{~b}$ & $1.17 \llbracket 0.04 \mathrm{c}$ & $17.81 \bowtie 0.01 \mathrm{~d}$ & $18.10 \llbracket 0.04 \mathrm{~d}$ \\
\hline $\mathrm{T}_{5}$ & $1.27 \bowtie 0.02 \mathrm{ab}$ & $1.29 \llbracket 0.04 \mathrm{ab}$ & $20.23 \llbracket 0.01 \mathrm{a}$ & $21.22 \varangle 0.01 \mathrm{a}$ \\
\hline $\mathrm{T}_{6}$ & $1.28 \llbracket 0.02 \mathrm{a}$ & $1.33 \llbracket 0.04 \mathrm{a}$ & $19.18 \bowtie 0.07 \mathrm{~d}$ & $18.19 \bowtie 0.04 \mathrm{~d}$ \\
\hline
\end{tabular}

Note: values are mean+ sd

\section{Total catechins content}

Catechin represents the unfermented product of black tea remained unchanged during the fermentation. The amount of total catechin holds prime importance for health enhancing properties of tea. Data representing the methanolic extract of $10 \mathrm{~min}$ extraction time in Table 4 indicating the peak value for this trait $\mathrm{T} 2$ as $1.30 \%$ followed by (1.29\%) in T3 while lowest recorded in T6 (TB2) as $1.27 \%$. Data regarding 20min extraction time disclosed the highest value of (1.36\%) in T1 (CB1) whereas lowest noted for the T5 (TB1) as $1.30 \%$. The increment of catechins was observed in the infusion of different samples of black and green tea extracts with solvents like methanol ethanol and acetonitrile as compared to water [26]. Enhanced extraction yield was obtained when extraction time was increased that revealed in the data represented (Table 5). The maximum catechin content in $\mathrm{T} 1$ represented its low degree of fermentation, which are responsible for its less thearubingins and color which is a marker of its quality. When the time of extraction increase by the same solvent the extraction efficiency was also increased for tea catechins [28].

\section{Caffeine}

Caffeine (3,7-dihydro-1,3,7-trimethyl-1H-purine-2,6 dione) belongs to a group of compounds collectively known as purine alkaloids [20,21]. Data regarding caffeine contents for extracts (Table 5) revealed that T6 (TB1) showed the highest caffeine content as 1.28 whilst the least caffeine contents were in T2 (CB2) sample $1.20 \%$. Extracts for 20 minutes showed maximum caffeine yield in T2 (1.34\%) and T3 exhibited minimum (1.17\%). In the instant study caffeine content of different tea brands were in the range of $1.22-1.25 \mathrm{~g} / 100 \mathrm{~g}$ liquid tea, are supported by the range of $1.09-1.65 \%$ as reported by [29]. It has been reported the mean percentage of caffeine in different tea samples is in the range of 1.011.19. Moreover, it was also proposed by [30] that the percentage of caffeine in tea is less than $1.50 \%$, indicating that Pakistani tea samples are risk free as this trait is below the threatening level.

\section{Conclusion}

Black tea phytochemicals that contributed towards its cup quality in Pakistan are in close proximity with international market. Methanolic extracts exhibited the more yield as a function of time. Cup quality of T1 was greater among the others while, tea bag of same brands showed fewer amounts of phytochemicals due to their permeability. Difference between the key quality parameters might be due to the processing technology, fermentation and storage period [31-35]. The result of recent investigation indicates that methanol provide a mean for optimization of cup quality, may be used as one of the quality tools for tea processing and marketing. It is concluded that investigated commercial brands were safe as for as their composition is concerned. However, safety evaluation of these methanolic extracts will be carried out by animal modeling.

\section{References}

1. Di W, Ying Z, Xiao L, Shuang W, Rong X, et al. (2011) Pu-erh black tea supplementation decreases quinocetone-induced ROS generation and oxidative DNA damage in Balb/c mice. Food and Chemical Toxicology 49(2): 477-484.

2. Almajano MP, Carboa R, Jimenez JAL, Michael H (2008) Antioxidant and antimicrobial activities of tea infusion Gordon. Food Chem 108: 55-63.

3. Espín JC, MT García Conesa, FA Tomás Barberán (2007) Nutraceuticals: facts and fiction. Phytochemistry 68(22): 2986-3008. 
4. Amić D, Lučić B (2010) Reliability of bond dissociation enthalpy calculated by the PM6 method and experimental TEAC values in antiradical QSAR of flavonoids. Bioorg 358 Med Chem 18(1): 28-35.

5. Cavia SM, Busto MD, Pilar Izquierdo MC, Ortega N, Perez Mateos M, et al. (2010) Antioxidant properties, radical scavenging activity and biomolecule protection capacity of flavonoid naringenin and its glycoside naringin, a comparative study. J Sci Food Agric 90(7): 1238-1244.

6. Das SA, Mukherjee M, Das D, Mitra C (2009) Protective action of aqueous black tea (Camellia sinensis) extract (BTE) against ovariectomy-induced oxidative stress of mononuclear cells and its associated progression of bone loss. Phytother Res 23(9): 1287-1294.

7. Ares G, Giménez A, Gámbaro A (2009) Consumer perceived healthiness and willingness to try functional milk desserts. Influence of ingredient, ingredient name and health claim. Food Qual Prefer 20(1): 50-56.

8. Gupta J, Siddique YH, Beg T, Ara G, Afzal M (2009) Protective role of green tea extract against genotoxic damage induced by anabolic steroids in cultured human lymphocytes. Biology and Medicine 1(2): 87-99.

9. Zhu F, Ratchaneekorn S, Sunan W (2016) Effect of black tea on antioxidant, textural, and sensory properties of Chinese steamed bread. Food Chem 194: 1217-1223.

10. Jayasekera A, Molan L, Garg M, Moughan PJ (2011) Variation in antioxidant potential and total polyphenol content of fresh and fullyfermented Sri Lankan tea Food Chemistry. 125(2): 536-541.

11. Jayasekera S, Molan AL, Garg M, Moughan PJ (2011) Variation in antioxidant potential and total polyphenol content of fresh and fullyfermented Sri Lankan tea. Food Chem 125(2): 536-541.

12. Schwager J, Mohajeri MH, Fowler A, Weber P (2008) Challenges in discovering bioactives for the food industry. Curr Opin Biotechnol 19(2): 66-72.

13. Macedo JA, Battestin V, Ribeiro ML, Macedo GA (2011) Increasing the antioxidant power of tea extracts by biotransformation of polyphenol a Food Chemistry 126(2): 491-497.

14. Rusak G, Likic S, Kovac M (2008) Phenolic content and antioxidant capacity of green and white tea extracts. Journal of food chemistry 110(4): 852-858.

15. Guo L, Jiacia G, Z Wencheng, Xinru J (2016) Optimized synchronous extraction process of tea polyphenols and polysaccharides from Huaguoshan Yunwu tea and their antioxidant activities. Food Bioproducts Processing 100: 303-310.

16. Sun C, Fu J, Chen J, Jiang L, Pan Y (2010) On-line HPLC method for screening of antioxidants against superoxide anion radical from complex mixtures. Journal of science 33(8): 1018-1023.

17. Belay A, Ture M Redi K, Asfaw A (2008) Measurement of caffeine in coffee beans with UV/vis spectrometer. Food Chemistry 108(1): 310315.

18. Brand Williams W, Cuvelier ME, Berset C (1995) Use of a free radical method to evaluate antioxidant activity. Lebensm Wiss Technolology 28: 25-30.

19. Steel RGD, Torrie, Dicky M JH (1997) Principles and Procedures of Statistics. Mc Graw Hill, New York, USA .
20. Awika JM, Rooney LW, Wu X, Prior RL, Cisneros Zevallos L (2003) Screening methods to measure antioxidant activity of sorghum (Sorghum bicolor) and sorghum products. Journal of Agriculture Food Chemistry 51(23): 6657-6662.

21. Yang CS, Wang X, Lu G, Picinich SC (2009) Cancer prevention by tea: animal studies, molecular mechanisms and human relevance. Nat Rev Cancer 9(6): 429-439.

22. Mphangwe N, Nyirenda LK (1997) Screening for quality potential in tea clones by chlorophyll fluorescence. Quarterly Newsletter. Tea Research Foundation (Central Africa) 125: 49-52.

23. Atoui AK, Mansouri AG, Boskou K, Kefalas P (2005) Tea and herbal infusions: Their antioxidant activity and phenolic profile. Food Chemistry 89(1): 27-36.

24. Auvichayapat P, Prapochanung M, Tunkamnerdthai O, Sripanidkulchai BO, Auvichayapat N, et al. (2008) Effectiveness of green tea on weight reduction in obese Thais: A randomized, controlled trial. Physiol Behav 93(3): 486-491.

25. Bai HW, Shim JY, Yu J, Zhu BT (2007) Biochemical and molecular modeling studies of the 0-methylation of various endogenous and exogenous catechol substrates catalyzed by recombinant human soluble and membrane-bound catechol-O-methyltransferases. Chem Res Toxicol 20(10): 1409-1425.

26. Quan PT, Hang TV, Ha NH (2007) Total polyphenols total catechins contents and DPPH free radical activity of several type of Vietnam commercial green teas. science and technology development 10(10): 11.

27. Obanda M, Owuor PO, Mangoka R, Kavoi MM (2004) Changes in thearubigins fractions and theaflavins levels due tovariations in processing conditions and their influence on black tea liquor brightness and total colour. Food Chemistry 85(2): 163-173.

28. Moisyadi S (1998) Cloning and characterization of a cDNA encodingxanthosine-N7-methyltransferase from coffee (Coffea arabica). Acta Hortic. 461, 15HB 367-d Agriculture. 82: 517-525.

29. Ayumiko N, Sumikotsuji Y, Tonogai (2003) Anaylsis of proanthocyanidinsin grapes seed extracts. Health foods and grapes seed oil. Journal of health science 49(1): 45-54.

30. Fujioka K, Shibamoto T (2008) Chlorogenic acid and caffeine contents in various commercial brewed coffees. Food Chemistry 106(1): 217-221.

31. Illy E (2008) The complexity of coffee. Scientific America 286: 86-91.

32. Bech Larsen T, Scholderer J (2007) Functional foods in Europe: Consumer research, market experiences and regulatory aspects. Trends Food Sci Technol 18(4): 231-234

33. Singleton VL, Orthofer R, RM Lamuela Raventos (1999) Analysis of total phenols and other oxidation substrates and antioxidants by means of Folin-Ciocalteu reagent. Enzymology 299: 152-178.

34. Tapsell LC, Hemphill I, Cobiac L, Patch CS, Sullivan DR, et al. (2006) Health benefits of herbs and spices: the past, the present, the future. Med J Aust 185(4): 4-24.

35. Xiao W, Yang Z, Chongxin F, Lujia H (2017) A method for producing superfine black tea powder with enhanced infusion and dispersion property. Food Chemistry 214: 242-247. 


\section{ISSN: 2574-1241}

DOI: $10.26717 / B J S T R .2018 .10 .001956$

AliImran. Biomed J Sci \& Tech Res

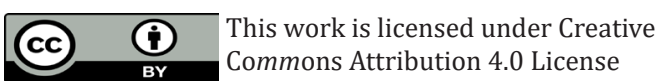

Submission Link: https://biomedres.us/submit-manuscript.php

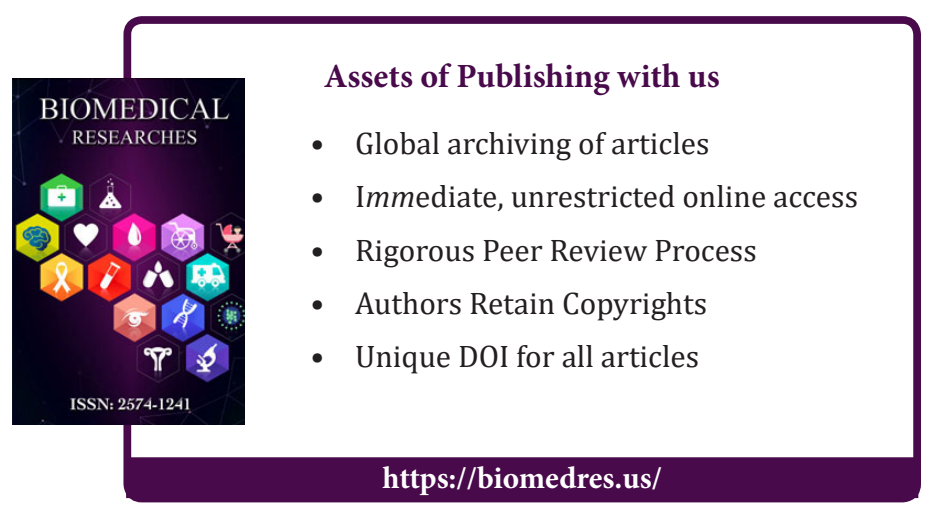

